Journal of research in health science

2018 № 1 (2), Januar-April

www.journalofresearch.org; info@journalofresearch.org

DOI $10.26739 / 2523-1243$

JOURNAL OF RESEARCH

INN HEALTH SCIENCE

ISSN Print: 2523-1243; ISSN Online: 2523-1251

\title{
The organization of medical care for epilepsy in pregnant women
}

\author{
Nilufar Rashidova \\ Department of Neurology, Tashkent Medical Academy, Tashkent, Uzbekistan.
}

Email address:

author.uzb@mail.ru (Nilufar Rashidova)

To cite this article:

Nilufar Rashidova. The organization of medical care for epilepsy in pregnant women. Journal of research in health science. Vol. 1, No. 2, 2018, pp. 52-55. DOI 10.26739/2523-1243

\section{dof http://dx.doi.org/10.26739/2523-1243/-2017-1-2-8}

\begin{abstract}
The issues of reproductive health of women with epilepsy are a significant problem of modern medicine. The proper management of women before and after pregnancy is an important step in preventing complications of the postpartum period, like woman with epilepsy and fetus. Treatment of women with epilepsy during pregnancy requires a special individual approach. Management of women with epilepsy of fertile age has its own characteristics and tactics, which must be taken into account individually with each patient.
\end{abstract}

Keywords: epilepsy, women, pregnancy, treatment, antiepileptic drugs.

\section{INTRODUCTION}

Epilepsy is a chronic brain disease characterized by repeated unprovoked seizures of motor, sensitive, vegetative, mental and mental functions caused by excessive neuronal discharges [1].

The incidence of epilepsy is $50-70$ cases per 100,000 people; prevalence is $5-10$ per 1000 people [1]. In Russia, the prevalence ranges from 1.1 to 8.9 cases per 1000 people. The risk of developing the disease depends on age, so the incidence of the disease in children under the age of 15 is often worse than among older persons - over 65 years of age [2].
Among the adult contingent of patients, about half are women of reproductive age, which is about $40 \%$ of women of childbearing age. Reproductive health of women with epilepsy is an urgent problem. This problem went beyond the medical framework, since in recent years the issue of qualitative and quantitative production of the population has become more acute [3]. Improving the reproductive health of the nation is one of the most important tasks of the state in the field of social policy.

Epilepsy, as one of the most stigmatizing diseases, significantly 
complicates the prospect of marriage and the creation of a family, and the most important issue for every woman - the possibility of having a child - causes a severe psycho-traumatic situation $[4,5]$.

At present, due to progress in epileptology and clinical pharmacology, it is possible to achieve a stable remission of the disease in more than $65-70 \%$ of patients, which can significantly improve their quality of life and social adaptation, to marry and have children. In connection with this, the problems of reproduction and the provision of therapeutic and diagnostic assistance to women with epilepsy have recently been particularly relevant [6].

Women aged 25-39 have significantly lower fertility rates than in the general population [7]. At the same time over the past 20 years, there has been a fourfold increase in the number of pregnancies and births in women with epilepsy [Vlasov P.N. et al, 2000; 2005]. Annually about 3 $(0.7 \%)$ of newborns from the total number of children are born from mothers with epilepsy [8,9].

More than $40 \%$ of women with epilepsy do not plan pregnancy [10]. Only $3 \%$ of women with epilepsy are planning to visit an obstetrician-gynecologist 3 months before the antici pated time of her onset. In addition, more than a third of women with epilepsy have no idea about the effect of pregnancy on the course of the underlying disease, although it is well known fact that the onset pregnancy in some cases can worsen the course of the disease [12].

The WHO experts have identified a list of diseases and conditions associated with a high risk of complications in case of an unplanned pregnancy, even life threatening and health threatening. It includes epilepsy [13].

Modern medicine has made considerable progress with regard to diagnosis and treatment of epilepsy. To date, the organization of rendering medical and social assistance to the patients with epilepsy is improved based on existing facilities. First of all, treatment and prophylactic institutions that have the capabilities of such high-tech research methods as MRI and CT of the brain, computer EEG (including EEG video monitoring), MR-angiography or selective angiography of cerebral vessels, therapeutic pharmacologic monitoring. Improving the organization of care for the patients with epilepsy and epileptic syndromes in the region makes it possible to optimize the prognosis of the disease and improve the quality of life of patients with epilepsy.

In connection with this, the optimization of therapy for epilepsy in women before pregnancy is important and consists in achieving remission of epilepsy at the lowest possible doses of antiepileptic drugs (AED) and preferably on monotherapy [14]. According to the recommendations of the Russian antiepileptic league, any replacement of AED should be avoided when remission is achieved [15]. One of the important moments in the treatment of epilepsy in pregnant women is the balanced and integrated approach of the treating doctor of the neurologist-epileptologist, obstetrician-gynecologist, with the aim of maintaining a balance between the risk for the mother and future fetus associated with uncontrolled seizures and teratogenic effects of AED [16].

Do rates of epileptic seizures linked with trimesters of pregnancy 
Nilufar Rashidova.

The organization of medical care for epilepsy in pregnant women.

The frequency of epileptic seizures during pregnancy does not change in most cases - 63.6-70.5\% of cases of women [17]. According to the literature, an increase in the frequency of seizures during the second trimester is observed in $32 \%$ of cases, in III - in $39 \%$. In $1.7 \%$ of cases, the frequency of seizures changes opposite to the original (increase or decrease, or, conversely) in the II and III trimesters compared with I trimester.

According to D. Battino, T. Tomson, E. Bonizzoni, 2013, worsening of seizure control in the II and III trimesters (compared to the ? trimester) is more common in pregnancy with lamotrigine therapy - at $19.9 \%$ than in those, who took carbamazepine $(14.6 \%)$, phenobarbital $(11.7 \%)$ or valproate (13.2\%).

Epileptic seizures are more likely in the first trimester of pregnancy with an increase in the drug load (in 35\% of cases with an increase in dose and / or the addition of a second / third AED) than without an increase in AED load (15.3\%) [18].

Is there any evidence association between estrogen and progesterone levels and convulsive epileptic seizures?

An increase in the level of estrogen in the first trimester of pregnancy and before childbirth can reduce the level of convulsive threshold [19]. In the period of gestation the increased estrogen content increases liver synthesis of a number of substances including procoagulants, fatty acids and steroids precursors, and the activity of liver cytochrome P450 increases [20]. Progesterone decreases intestinal motility, preventing the secretion of mucous secretion, the $\mathrm{pH}$ of gastric juice and can affect the absorption of AED [21]. Due to these processes, the dose of AED increases during pregnancy in $26.0 \%$ of cases, the addition of second AED with initial monotherapy is observed in $2.6 \%$ of all pregnancies in women with epilepsy. The increase in the dose and / or the addition of another AED was recorded in $16.7 \%$ of cases with the initial monotherapy with carbamazepine, $47.7 \%$ with lamotrigine, $29.3 \%$ with phenobarbital and $20.5 \%$ with valproate [18]. In the postpartum period, the level of most AEDs in the mother's blood gradually increases and reaches its initial value at the tenth week after delivery [21].

\section{Conclusion}

Summarizing the foregoing, the management of women with epilepsy of fertile age has its own characteristics and tactics, which must be taken into account individually with each patient.

\section{References}

1. Klotz, U. The role of pharmacogenetics in the metabolism of antiepileptic drugs: pharmacokinetic and therapeutic implications / U. Kloz // Clin. Pharmacokinet. - 2007.-Vol. 46.-P. 271-279.

2. Pregnancy, delivery, and outcome for the child in maternal epilepsy / G. Veiby, K. Daltveit, B. A. Engelsen [et al.] // Epilepsia. - 2009. - Vol. 50, № 9. - P.2130-2139.

3. Choice of method of contraception in women suffering from epilepsy / LV Adamyan, I.A. Jidkova, V.V. Berseneva and others // Pharmateka - 2012. - № 4.-With. 14-19.

4. Analysis of prenatal and gestational care given to women with epilepsy /C. Seale, M. Morrell, L. Nelson [et al.] // Neurology. - 1998. - Vol. 51, № 4. - P.1039-1045. 
Journal of research in health science. 2018; 1 (2): 52-55.

5. Best practice guidelines for the management of women with epilepsy / P. Crawford, R. Appelton, T. Betts [et al.] // Seizure. - 1999. - Vol. 8, № 4. - P.201-217.

6. Vlasov, P.N. Therapy of epilepsy in preparation for pregnancy and its management // P.N. Vlasov, V.A. Petrukhin // Modern therapy in psychiatry and neurology. - 2013. - No. 3-4. - P. 32-38.

7. Vlasov, P.N. Pregnancy in epilepsy, problems and prospects. N. Vlasov // Epilepsy and paroxysmal conditions. - 2011. - No. 4. P.45-46.

8. Lepakhin, V.K. Methods for detecting and recording adverse reactions to medications during their widespread use / V.K. Lepakhin, N.V. Sturov, A.V. Astakhova // Difficult patient. - 2008. - No. 6. - P. 42-48.

9. Seizure control and treatment changes in pregnancy: Observations from the EURAP epilepsy pregnancy registry / D. Battino, T. Tomson, E. Bonizzoni [et al.] // Epilepsia. - 2013. - Vol. 54, № 9. - P. 1621-1627. 10. Seizures control in anti-epileptic drug-treated pregnancy / F. J. Vajda, Hitchcock, J. Graham [et al.] // Epilepsia. - 2008. - Vol. 49, № 1. - P.172-175.

11. Morrell, M. J. Epilepsy in women: the science of why it is special / M. J. Morrell //Neurology. - 1999. - Vol. 53, Suppl. 1. - P. 42-48.

12. Pennell, P. B. Antiepileptic drug pharmacokinetics during pregnancy and lactation / P. B. Pennell / / Neurology. - 2003. - Vol. 61, № 6 (Suppl. 2). - P. 35-42.

13. Loebstein, R. Pharmacokinetic changes during pregnancy and their clinical relevance / R. Loebstein, A. Lalkin, G. Koren // Clin. Pharmacokinet. - 1997. - Vol. 33, № 5.-P. 328-343.

14. Tomson, T. Pharmacokinetics and therapeutic drug monitoring of newer antiepileptic drugs during pregnancy and the puerperium / T. Tomson, D. Battino // Clin. Pharm. - 2007. - Vol. 46, № 3. - P. 209-219. 15. V.V. Karlov. Epilepsy: diagnosis and treatment. M. Medicine: 2004. 4.7: 30-34.

16. Vlasov P.N.Pharmaco-hormonal relationships in the use of carbamazepine and valproic acid in patients with epilepsy of women of childbearing age. Journal of neuropathology and psychiatrist 2001; 11: 26-30.

17. Odintsova, G.V. Social aspects of female epilepsy // G.V. Odintsova, J1. A. Saykova // SOCIS (Sociological Research). - 2012. - № 3. -C. 116-119.

18. Hormonal contraception in high-risk women: solutions problems / EA Mejevitinova, M.B. Khamoshina, O.D. Rudneva and others. Doctor.Ru. - 2012.-№ 1. - P. 27-33.

19. Djeleznova, E.V. Quality of life and social functioning of women with epilepsy / E.V. Djeleznova / / Proceedings of the Congress "The Man and the Medicine." - M., 2005. - P.10-11.

20. Mikhailov, V.A. Epilepsy in Women: Socio-Psychological Aspect, Quality of Life, Family Rehabilitation Issues / V.A. Mikhailov, S.A. Gromov, E.S. Eroshina // Jour. Neurology and psychiatry. S. S. Korsakov. - 2007. - No. 10. - P. 61-64.

21. The state of medical and social care for patients with epilepsy and their families in Jeleznogorsk / / G.Ya. Melnikov, A.V. Sadykova, N.A. Schneider and others // Herald Clinical Hospital No. 51. 2008.-No. 2. - P. 51-55. 